\title{
Ontotecnia, ingeniería organizacional y actores emergentes
}

\section{Ontotechnics, organizational engineering and emerging actors}

\author{
Alejandro Ochoa Arias y Juan Antonio González de Requena Farré \\ Universidad Austral de Chile
}

\begin{abstract}
Resumen
En el pensamiento contemporáneo se ha producido cierto desplazamiento desde la comprensión ontológica tradicional de la tecnología, y se perfila una auténtica ontotecnia, la cual se hace cargo de las formas de hibridación socio-natural y tecnocultural. Las formas de anticipación y repetición propias de esa deriva ontotécnica se caracterizan por un efecto espectral de desfase y obsolescencia, pero también de investidura imaginaria de los actores tecno-culturales. En este artículo, se exploran los isomorfismos que existen entre las metamorfosis de los objetos técnicos y la emergencia espectral de los actores sociopolíticos. Esta indagación se lleva a cabo en particular en el desarrollo de la Investigación de Operaciones Comunitaria y en el desarrollo de las iniciativas de la Tecnología Libre, para explorar su significado en el presente.
\end{abstract}

Palabras clave: ontotecnia, espectralidad, historia de la tecnología, actores políticos.

\begin{abstract}
In contemporary thought there has been a certain shift from traditional ontological understanding of technology, and it is being sketched a real ontotechnics that takes charge of the forms of socionatural and techno-cultural hybridization. The forms of anticipation and repetition of this ontotechnical drift are characterized by a spectral effect of mismatch and obsolescence, but also of imaginary investment of techno-cultural actors. In this article, we explore the isomorphism between that metamorphosis of technical objects and the spectral irruption of sociopolitical actors. This survey considers the development of Community Operational Research and Free Technology, in order to explore their meanings at the present.
\end{abstract}

Keywords: onto-technics, spectrality, history of technology, political actors.

Recibido: 8-1-18. Aceptado: 15-4-18.

Alejandro Ochoa es Doctor en PhD. Systems and Management Sciences. Juan Antonio González de Requena es Doctor en Filosofía. Ambos trabajan como profesores en la Universidad Austral de Chile.

Contacto: A. Ochoa, Universidad Austral de Chile, Sede Puerto Montt Los Pinos S/N. dioseses@gmail.com

J. A González de Requena, Universidad Austral de Chile, Sede Puerto Montt Los Pinos S/N. jagref8@gmail.com

Cómo citar: Ochoa Arias, A., y González de Requena Farré, J. A. (2018). Ontotecnia, ingeniería organizacional y actores emergentes. Revista Stultifera, 1 (1), 83-109. ISSN 0719-983X 


\section{ONTOTECNIA, INGENIERÍA ORGANIZACIONAL Y ACTORES EMERGENTES}

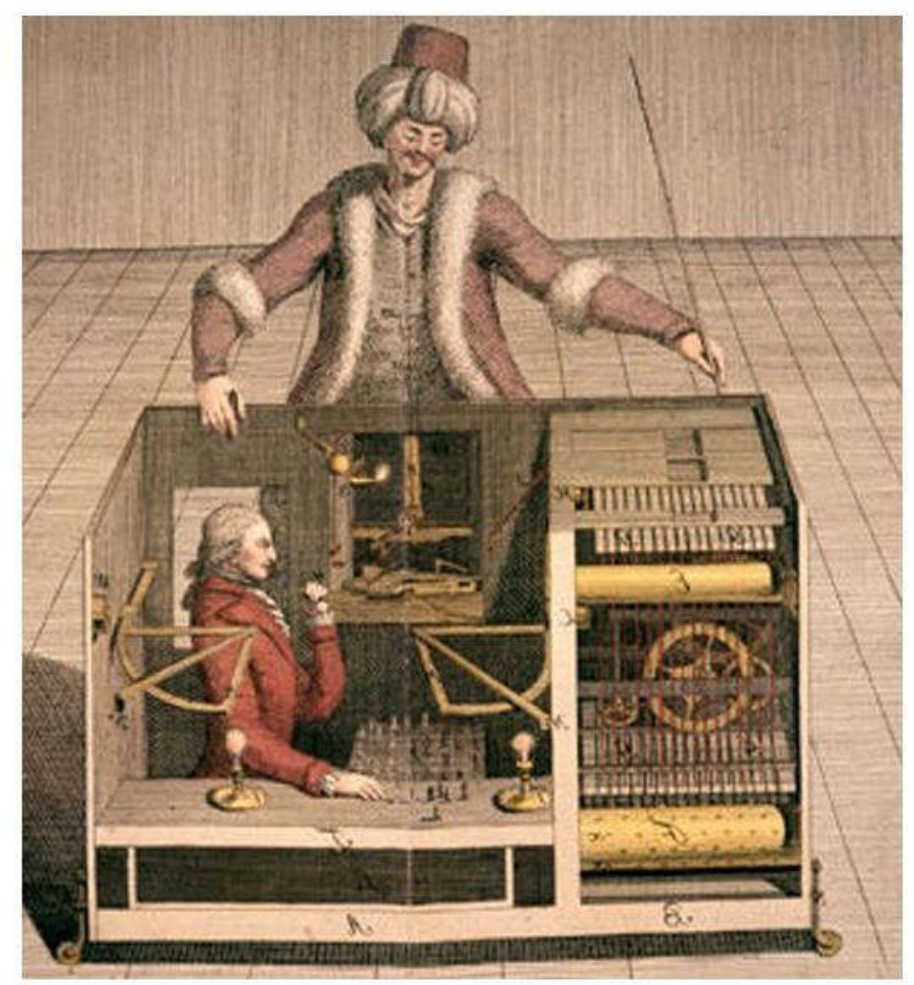

Figura 1. El truco del ajedrecista turco. Fuente: http://www.juguetessomosnosotros.com/wpcontent/uploads/2014/03/EL-TURCO-TRUCO.jpg

En 1769, el supuesto barón Wolfgang von Kempelen construyó uno de los autómatas que más fascinación han generado en nuestro imaginario cultural: se trataba de un jugador de ajedrez turco, sentado ante el tablero dispuesto sobre un mueble aparentemente repleto de resortes mecánicos; jugaba al ajedrez con tal destreza que era capaz de ganarle partidas a intelectos tan connotados como Benjamin Franklin o Napoleón (véase la figura 1). El jugador de ajedrez automático cambió de dueño y operador durante sus ochenta y cinco años en funciones, de manera que también es conocido como el jugador de ajedrez de Maelzel, uno de los propietarios, quien montó un espectáculo con el turco, exitosamente exhibido por el viejo y el nuevo continente. Era tal el interés por develar el enigma del funcionamiento del turco jugador de ajedrez que se publicaron numerosos textos acerca del asunto, entre los cuales destaca el ensayo de Edgar Allan Poe (1973). Este concluyó que, en el seno del artefacto, actuaba en realidad un ajedrecista humano, ingeniosamente oculto mediante juegos de espejos y compartimentos internos. 


\section{ALEJANDRO OCHOA Y JUAN ANTONIO GONZÁLEZ DE REQUENA}

Lo que el crítico buscaba dentro del autómata era un fantasma en la máquina, en el más pleno sentido del término: se trataba de conjurar una inteligencia humana, misteriosamente velada por el operador y distinta de este, que diera cuenta de las operaciones del ajedrecista turco. La apelación a un personaje escondido en el mecanismo era la manera más razonable de explicar las irregularidades e imprecisiones del autómata. El autómata jugador de ajedrez siguió ejerciendo su fantasmático influjo sobre el imaginario cultural contemporáneo, de manera que, cuando nos hacemos cargo del problema de la técnica, frecuentemente se mantiene la pregunta sobre quién es el personaje oculto moviendo el mecanismo, y conjuramos al actor escondido en la máquina.

¿Resulta posible abordar el asunto de la técnica sin conjurar el fantasma en la máquina? ¿Es posible, siquiera, pensar filosóficamente (esto es, emprender esa búsqueda siempre ya desfasada - de un saber perdido de antemano y aventurarse en ese vuelo intelectual crepuscular que intenta concebir con cierto retraso lo que ya está en despliegue) y no conjurar alguna rémora espectral en el intento de concebir la actualidad? No en vano, se podría pensar que un saber aparentemente tan desfasado como el de la filosofía no puede dar cuenta de lo emergente. Sobre todo, si se trata de aprehender el sentido de las nuevas configuraciones socio-técnicas que se esbozan en la trama de nuestras formas de vida. No en vano, la filosofía parece haber apostado desde sus orígenes por un repudio sistemático de toda técnica que pretendiera suplantar la presencia viviente, y ha denostado la reproducción artificial de copias repetibles, empezando por la tecnificación del discurso en la retórica. Aun así, invocaremos el espectro de la filosofía, toda vez que se trata de llevar a cabo una tarea eminentemente espectral, a saber: conjurar los fantasmas de los actores emergentes que se esbozan en las nuevas redes socio-técnicas.

\section{De la ontoteología, a la ontotecnia}

Ciertamente, el asunto del pensar filosófico tradicional cae en el rubro de una ontoteología que se hace cargo de la pregunta por el ser de un modo muy específico, a saber: como una definición esencial de los entes que privilegia su presencia estable; como una aproximación representativa del ser de los entes que trata de acotar su identidad en el marco estructurador del juicio lógico, y que reduce la verdad a la corrección o adecuación lógica; o como una indagación de los entes, que sustenta el ser condicionado de las cosas en algún 


\section{ONTOTECNIA, INGENIERÍA ORGANIZACIONAL Y ACTORES EMERGENTES}

fundamento último o ser supremo incondicionado (Heidegger, 1990; 1991; 1996). Desde esta perspectiva metafísica del ser de los entes, que privilegia la disposición y aseguramiento de su presencia a partir de algún fundamento incuestionable, la técnica no puede aparecer sino como un modo subordinado del saber, un saber contingente y sin fundamento, sostenido en la repetición mecánica, y conducente a un artefacto que resulta externo al principio de su fabricación.

Ello no impedirá que la ontoteología tome prestados sus paradigmas de las técnicas más diversas y de diferentes modos de fabricación: el modelado, la acuñación, el pilotaje, el tejido, la arquitectura, la escritura, la relojería, la mecánica hidráulica, etc. No en vano, en la modernidad se producirá una alianza manifiesta entre las artes y los saberes teóricos, que hasta el medievo habían estado jerárquicamente disociados, y de esa conjugación emergerán tanto las artes científicas como la ciencia experimental y técnicamente orientada. Es más, podríamos sospechar que la técnica, la disposición de los objetos para su control a escala planetaria, constituyó el horizonte de la metafísica y la consumación de una ontoteología que ha proyectado precisamente el aseguramiento del ser como estructura estable y con fundamentos fijos. Al menos, en algunas de las figuras modernas de la ontoteología, el dominio y enseñoramiento sobre un mundo calculable es un propósito explícito del pensar filosófico, en tanto que el mecanismo y los autómatas de diferentes tipos se convierten en modelos explicativos que generan una extraña fascinación.

Ahora bien, el proyecto de destrucción de la ontoteología tradicional y la deriva postmetafísica de la autocomprensión filosófica en la modernidad tardía han puesto en jaque algunos de los presupuestos centrales de la ontoteología tradicional. Desde diferentes perspectivas, se ha impugnado la disposición cosificada de los entes como algo dado; se ha cuestionado el encuadramiento lógico del pensar bajo el orden de la representación objetivadora, y se ha atacado el fundamentalismo metafísico y la pretensión de que solo resulta posible pensar con validez desde fundamentos incontrovertibles, más allá de la contingencia y el devenir. En ese sentido, cabe reconocer cierta revolución ontológica en el pensar filosófico contemporáneo, que apela a una comprensión más originaria, existencial, encarnada y arraigada de nuestro ser en el mundo. Se asume, así, como tarea la interpretación participante de los modos de hacer sentido del ser a través de nuestra actividad involucrada, 


\section{ALEJANDRO OCHOA Y JUAN ANTONIO GONZÁLEZ DE REQUENA}

elucidando el trasfondo de nuestras tradiciones y prácticas compartidas; y, de esa manera, se promueve un acercamiento discursivo y contextual al sentido del ser, reconociendo abiertamente su contingencia, facticidad histórica y oscilación interpretativa (Gadamer, 1995; Rorty, 1991; Vattimo, 1991).

No obstante, las ontologías contemporáneas han mostrado un particular recelo al hacerse cargo de la técnica. En la misma medida en que han apelado a nuestra capacidad para la apertura de horizontes de sentido desde el trasfondo de tradiciones compartidas, han considerado que la técnica constituye un modo extrañado de involucrarse en el mundo, basado primordialmente en la pro-vocación orientada al control, la planificación coactiva, la reproducción programada y el dispositivo calculable. Aunque se han alejado de la visión de la técnica como instrumento y han entendido el quehacer técnico como una manera de posicionarse en el mundo y desocultar lo real, las ontologías contemporáneas terminan asociando la técnica a la racionalización instrumental y la disposición calculable que permite explotar y acumular recursos (Heidegger, 1994). El referente de esta visión de la técnica se encuentra en la industria tecnológica y en la ingeniería industrial, desde la central hidroeléctrica y la explotación de la energía atómica, hasta la agroindustria motorizada y la informatización del lenguaje. En fin, el privilegio concedido en las ontologías contemporáneas al decir dialógico y al pensar poetizante $-\mathrm{y}$, en última instancia, al logos discursivo-, en tanto que modos eminentes de comprensión auténtica y apertura del sentido, se traduce en cierta incapacidad para dar cuenta de los entornos técnicos y del funcionamiento de los sistemas expertos.

A la zaga de las mutaciones tecno-culturales contemporáneas, en el pensamiento de las últimas décadas se viene esbozando una auténtica ontotecnia, más allá de las pretensiones de sentido y de las formas de comprensión centradas en el discurso humano que han caracterizado a la ontología. Se trata de una exploración de la capacidad de transformación funcional y de síntesis operacional de los entornos técnicos; en ese sentido, la ontotecnia reivindica a la técnica en cuanto ámbito fundamental de construcción y organización de las relaciones socio-naturales. Ciertamente, en el contexto de la ontotecnia son desbordadas algunas categorías fundacionales de la metafísica occidental. La concepción metafísica del individuo dotado de una esencia fija y una unidad fundante da paso a una consideración del 


\section{ONTOTECNIA, INGENIERÍA ORGANIZACIONAL Y ACTORES EMERGENTES}

complejo proceso de individuación que da lugar a la génesis de los objetos técnicos, a través de las transformaciones operacionales, los acoplamientos funcionales y los nexos transindividuales; al fin y al cabo, el objeto técnico no tiene más sustancia individual que su deriva morfo-técnica (Simondon, 2007).

Asimismo, la perspectiva ontotécnica desbarata algunas de las oposiciones subordinantes que han fundado la metafísica occidental; y es que las distinciones entre materia y forma, sujeto y objeto, natural y artificial, naturaleza y sociedad, teoría y práctica, o técnica y ética, se desdibujan cuando consideramos las interrelaciones funcionales, las formas de mediación operacional y la composición híbrida de las redes tecno-culturales y los entornos tecno-naturales en los cuales nos desenvolvemos y a los cuales estamos acoplados. Este multiverso ontotécnico está constituido por híbridos socio-naturales y tecno-culturales en que lo no humano y lo social se entremezclan; por cuasi-objetos en que la construcción del artefacto no está separada de la cuestión de hecho (Latour, 2007); por redes transindividuales en que se transmiten y comunican singularidades (Serres, 1995); por cyborgs o híbridos de organismo y máquina, criaturas ontotécnicas e identidades cibernéticas que surgen del acoplamiento de lo natural, lo social y lo artificial (Haraway, 1991).

Por supuesto, el nexo operacional del objeto técnico no se identifica con su condición de útil, en la medida en que distintos conjuntos funcionales podrían suministrar una misma utilidad o rendimiento. El ámbito ontotécnico no se dejaría reducir en ningún caso a la utilización instrumental, con la cual nos ha familiarizado históricamente una alienante experiencia del trabajo en que solo se trata de la acumulación del producto a través de una relación de extrañamiento con la máquina (Simondon, 2007). Por el contrario, son las derivas operacionales de los conjuntos técnicos y las redes tecno-culturales las que, en gran medida, permiten dar cuenta de las transformaciones en los modos de producción y de las reubicaciones de las esferas públicas. Además, intervienen en la reorganización del sensorio y en las modificaciones de las matrices de recepción cultural, así como en las reestructuraciones de los medios de transmisión, de las redes de comunicación o de los regímenes de circulación y distribución de los signos (Benjamin, 1973; McLuhan, 1985). El referente de la construcción ontotécnica se halla en las nuevas tecnologías de la información y comunicación, desde los medios electrónicos hasta las redes telemáticas, así como en la 


\section{ALEJANDRO OCHOA Y JUAN ANTONIO GONZÁLEZ DE REQUENA}

biotecnología y la ingeniería genética o en las nuevas tecnologías organizacionales y en la ingeniería del comportamiento.

En suma, en una perspectiva ontotécnica, el modelo del instrumento (del medio de producción, del útil a la mano, o de la actividad instrumental) ya no resulta pertinente, pues presupone una comprensión de lo técnico demasiado humana, que contrapone y subordina la técnica al designio del usuario humano, e introduce, así, una versión de los entramados tecnoculturales tan antropológica como antropocéntrica. La construcción ontotécnica da cuenta del modo de existencia de los objetos técnicos en términos de las síntesis funcionales y convergencias operacionales que se organizan en una unidad estructural y en un conjunto causalmente recurrente en su medio. No se trata de la simple composición mecánica de elementos funcionales en un ensamblaje artificial, sino — como plantea Simondon- del desarrollo de una sinergia funcional y orgánica en una estructura auto-organizada; ello no excluye cierto margen de diferenciación de la estructura en subsistemas funcionales, aunque prima la estandarización interna del esquema de funcionamiento inherente a un plexo operacional estable. Tampoco se ha de confundir la existencia de los objetos técnicos con la automatización o el automatismo, pues la unificación estructural ontotécnica involucra una cierta indeterminación operacional y algún margen de ausencia de especialización funcional, en virtud de la cual el objeto técnico pueda acoplarse en otras series de operaciones e integrar nuevas síntesis funcionales (Simondon, 2007).

Desde el punto de vista ontotécnico, cabe afirmar, entonces, que además de los entes físicos inorgánicos y de los seres biológicos organizados irrumpe una categoría híbrida de entes inorgánicos organizados con sus propias derivas y acoplamientos funcionales autónomos. En ese sentido, la construcción ontotécnica responde al modelo protético, esto es, al suplemento que reinscribe aquello que extiende, y así el qué y el quién de la invención se tornan indiscernibles; sujeto y objeto (así como usuario y estructura funcional) de la invención técnica se distinguen únicamente sobre el trasfondo indecidible de una deriva de diferenciación dinámica de las funciones y a partir de cierta articulación pro-gramática de las sinergias operacionales (Stiegler, 2002). Cabe afirmar, pues, que la construcción ontotécnica se sostiene en algún medio que opera tanto sobre soportes, dispositivos, equipos y vectores técnicos, como sobre códigos, matrices y marcos institucionales; de esa manera, técnica y 


\section{ONTOTECNIA, INGENIERÍA ORGANIZACIONAL Y ACTORES EMERGENTES}

práctica, materia organizada y organización materializada se conjugan en los medios ontotécnicos (Debray, 1991).

Llega hasta tal punto la capacidad ontotécnica de extensión, transferencia y reorganización de nuestros modos de experiencia que puede atribuírsele una marcada productividad tropológica. Así, pues, las técnicas operan metafóricamente, al introducir desplazamientos y reestructuraciones en nuestras relaciones con los medios: no solo redefinen las relaciones entre figura y fondo en nuestros medios experienciales, sino que llevan a cabo una compleja interconexión entre aquello que cada construcción ontotécnica realza, aquello que deja obsoleto, aquello que recupera y aquello en que el medio técnico se transforma cuando es llevado hasta el límite de su potencial (McLuhan, 1996). No es de extrañar que finalmente se esté reivindicando el aspecto retórico de la reconstrucción ontotécnica, de modo que —en un curioso giro histórico-, se invierte el rechazo ontoteológico de la retórica como técnica por excelencia.

En virtud de su potencia de desplazamiento, transferencia y reorganización, la construcción ontotécnica introduce modos de retención y anticipación, de repetición y de invención, de inactualidad y de virtualidad, que descoyuntan la presencia del ser; la falta de sincronía del medio técnico, atravesado por las obsolescencias y las programaciones, expone la ausencia de contemporaneidad a sí del presente socio-técnico. En ese sentido, la construcción ontotécnica desborda toda ontoteología u ontología concebible; no solo destituye el discurso sobre la presencia del ser, sino que, además, incorpora una deriva espectralizadora, cierto efecto de espectralidad consistente en una temporalidad diferida, tan programática como fantasmática (Derrida, 1995).

No se trata únicamente de que todo intento de aprehender el logos del presente o la estructura categorial del ser recurra inevitablemente a categorías desfasadas por las propias transformaciones y derivas de aquello que se pretende concebir; ni nos referimos exclusivamente al hecho de que toda actividad introduce contingencias y efectos colaterales inanticipables que no solo desbordan cualquier cálculo de las dinámicas actuales, sino que acompañan como una sombra a las construcciones ontotécnicas. A la obsolescencia espectral del logos y al fantasma de los efectos incalculables, se suma que las consecuencias de toda construcción ontotécnica consisten precisamente en la construcción ontotécnica de las 


\section{ALEJANDRO OCHOA Y JUAN ANTONIO GONZÁLEZ DE REQUENA}

consecuencias. De ese modo, el desfase, la disyunción, la obsolescencia son efectos estructurales inherentes a la construcción ontotécnica, aunque toda una corte de metafísicos de la técnica, con vocación de futurólogos, se esmeren por establecer la configuración esencial de la tecnología actual; al fin y al cabo, la conjuración del fantasma, así como el reconocimiento imaginario y especular en el deseo proyectado, constituyen movimientos de una fantasmología posible y se inscriben en la dinámica de la espectralidad.

\section{La deriva ontotécnica}

La deriva ontotécnica está marcada por las convergencias operacionales y las sinergias funcionales; pero también resulta enmarcada por los desplazamientos tropológicos, la ausencia fantasmática y el anacronismo espectral. Ciertamente, se puede reconocer —con Simondon (2007) — un desarrollo ontotécnico guiado por un proceso de concretización, o sea por una creciente unificación estructural de los nexos operacionales, por la complejización de las sinergias funcionales y la organización autónoma del objeto técnico. Habríamos transitado, pues, desde el modo abstracto de los objetos técnicos (ése que privilegia el ensamblaje analítico de elementos funcionalmente autosuficientes en un sistema artificialmente cerrado), hasta la forma concreta del objeto técnico, caracterizada por la individualización sintética y la unificación estructural orgánica, por la elevada coherencia operacional y la autoorganización funcional, así como por la interconexión, traducibilidad y apertura a redes y conjuntos técnicos. Análogamente, se puede señalar que se ha pasado de una producción artesanal del objeto técnico (en que éste es fabricado a medida del usuario e incorpora sus condiciones y estándares del entorno social), a una producción industrial, en que el objeto técnico exhibe una coherencia funcional y un estándar interno, que le dan forma al sistema de necesidades sociales, así como conservan la individualidad del objeto técnico en la interconexión de las redes operacionales y los conjuntos técnicos (Simondon, 2007).

Por otra parte, existen distintos relatos que tratan de dar cuenta de algunos periodos y discontinuidades decisivas de la deriva ontotécnica. Desde la perspectiva de la historia de la tecnología, a veces se ha descrito la deriva ontotécnica en términos de las energías y materiales movilizados: se habría producido un desplazamiento desde una fase eotécnica (el complejo tecnológico que, entre el siglo X y el XVIII, se sustentó en la utilización de recursos naturales renovables como el agua y la madera), pasando por una fase paleotécnica 


\section{ONTOTECNIA, INGENIERÍA ORGANIZACIONAL Y ACTORES EMERGENTES}

(desarrollada entre el siglo XVIII y el XIX y que se basó en los combustibles no renovables como el carbón y en metales como el hierro), hasta alcanzar la actual fase neotécnica, que constituye un complejo tecnológico de materiales sintéticos, nuevas aleaciones y electricidad (Mumford, 1987). Pero no se trataría únicamente de una deriva técnica desde la utilización de energía cinética natural y animal, pasando por la energía termodinámica de las máquinas motorizadas, hasta la energía electromagnética que sostiene las nuevas redes tecnológicas; también se puede reconocer un tránsito desde una relación más exterior y mecánica con la máquina, hasta conjuntos tecnológicos más orgánicos, integrados y a escala humana, que incluso impulsan la ingeniería del comportamiento y las más diversas tecnologías organizacionales.

En otro sentido, cabe afirmar - con Levy (2004) que la deriva ontotécnica se caracteriza por ciertos desplazamientos en la escala de las tecnologías: las tecnologías arcaicas — basadas en el ensamblaje mecánico, la producción por cuerpos vivientes, la selección natural y la gran escala del paisaje natural - habrían sido reemplazadas por las tecnologías molares, desplegadas en conjuntos técnicos autosuficientes y que se basan en la producción y utilización motorizada de energía termodinámica, así como en la selección artificial de los usuarios. Por último, veríamos irrumpir toda una gama de tecnologías moleculares que minimizan la escala de la técnica, tal y como ocurre en la nanotecnología, la ingeniería genética o la digitalización informática (Levy, 2004).

Por cierto, también se ha descrito la deriva ontotécnica en términos de las transformaciones en la experiencia del trabajo que se le asocian (Serres, 1995): de la labor cíclica de dar forma a las cosas, de ese trabajo cinemático y hercúleo que transporta y sustenta (como ocurre en la labor del labrador, el tejedor, el arquitecto, el tallador o el navegante), se habría transitado a formas del trabajo termodinámicas, caracterizadas por la irreversible transformación prometeica basada en la potencia del fuego que opera en las máquinas motorizadas (como en la técnica industrial). En la actualidad estaría constituyéndose una modalidad mercurial del trabajo — más cercano al rol de mensajero de Hermes-, basada en la comunicación, transducción y transmisión, así como en la tarea de organización de la información en las redes informáticas y los microprocesadores digitales (Serres, 1995). 


\section{ALEJANDRO OCHOA Y JUAN ANTONIO GONZÁLEZ DE REQUENA}

\section{La Ingeniería de Organizaciones, desde la ontotecnia}

El planteamiento del desplazamiento del logos por la pragmática de una técnica del ser - que se plasma en la ontotecnia planteada en la sección anterior- implica que el ejercicio de las formas de concebir, diseñar e implantar en la sociedad los avances propios de las tecnologías tengan una potencia que va más allá de la pretendida por el discurso hegemónico del control y predicción de los fenómenos sobre los cuales se edifican las ciencias empíricas y el ejercicio sistemático de la transformación de la naturaleza, como la reconstrucción al infinito de la morada del ser humano en el mundo.

El destronamiento del discurso en la constitución y construcción del ser no es sustituido por el silencio porque eso supondría una ausencia del discurso que, en esencia, se mantiene en el plano del discurso, lo cual reafirmaría a la persuasión y el reconocimiento del otro como los modos sobre los cuales se define al ser. ¿Cuáles serían las implicaciones de esta noción de ontotecnia para la práctica de la ingeniería? ¿Cómo interpela o gobierna a la ingeniería que se ocupa esencialmente del diseño y puesta en marcha de los sistemas híbridos socio-naturales y tecno-culturales? Es decir, ¿cómo impacta en el proceso de gestión de la forma social más ampliamente difundida y regulada: las organizaciones?

A continuación, se explora una interpretación, desde una perspectiva ontotécnica, de dos planteamientos que son en principio contrarios a la instrumentalización basada en la tecnología. El primero está referido al uso de las técnicas y métodos de la investigación de operaciones en el ámbito comunitario, y la segunda se asocia con el concepto de tecnología libre y conocimiento libre como una forma contracultural de los procesos hegemónicos técnicos del presente.

Investigación de Operaciones Comunitaria: Manifestación de la ontotecnia o rémora de la ontoteología.

Desde el campo de la Investigación de Operaciones —área dedicada a la gestión racional de medios para la producción eficiente y que constituye uno de los factores que han dado lugar al desarrollo del pensamiento estratégico, la ingeniería de organizaciones y la tecnología de la organización desarrollada hasta el presente- se dio a finales de los años 80 y comienzos de los 90 un proceso de ampliar el acceso de las técnicas de modelado y predicción a las 


\section{ONTOTECNIA, INGENIERÍA ORGANIZACIONAL Y ACTORES EMERGENTES}

organizaciones comunitarias y, de forma más general, permitir el acceso a los sectores menos favorecidos de la sociedad a la racionalidad detrás del modelado de los problemas de gestión. El debate que se originó está documentado en varias publicaciones (ver Ritchie, Taket y Bryant, 1994; Midgley y Ochoa-Arias, 2004). Recientemente, la Investigación Comunitaria ha retomado la discusión de su sentido y propósito. Lo alcanzado como definición es importante porque permite identificar el centro de la discusión: la condición comunitaria.

En la trayectoria de este debate desde los años 90, el punto álgido ha estado centrado en poder caracterizar el objeto (¿o acaso será el sujeto?) de lo que se supone es una disciplina centrada en las herramientas de gestión organizacional. Este debate ha conducido a explorar las visiones de comunidad (Midgley y Ochoa-Arias, 2004) que se podían ver representadas en la práctica de esta rama de la Investigación de Operaciones. Esfuerzos similares han realizado (Johnson, 2012; Parry y Mingers, 1991), y los resultados arrojan la dificultad no solo de ponerse de acuerdo, sino además una cierta inconformidad en el afán de caracterizar al cliente, usuario o interlocutor al cual se entregan los instrumentos de gestión de operaciones para ayudarles a ser más eficientes no solo en la realización de sus propias tareas, sino además —y esto resulta quizás más importante para el propósito de este artículo— para poder tener elementos que le permitan conducirse de acuerdo a la racionalidad con la cual se toman las decisiones en los aparatos del estado y de las formas hegemónicas de la sociedad inglesa en su momento y, en el proceso de expansión, en cualquiera de las sociedades en la cual se desarrolla la práctica de la Investigación de Operaciones Comunitaria (IOC). Es decir, en los esfuerzos por difundir la práctica de la Investigación de Operaciones la propuesta comunitaria alimenta dos corrientes contrarias entre sí: una orientada a la ampliación de los usuarios de los métodos de esa disciplina (incorporando a las organizaciones comunitarias) y la otra preocupada por ampliar la reflexión sobre los procesos de toma de decisiones que se dan en ámbitos distintos a los usualmente incorporados en los modelos de empresas asociadas al proceso productivo tecnológico. Estas corrientes identificadas desde el comienzo mismo de su lanzamiento como propuesta (ver Rosenhead, 1996) han estado presentes en mayor o menor intensidad en las reflexiones asociadas a la Investigación de Operaciones Comunitaria. 


\section{ALEJANDRO OCHOA Y JUAN ANTONIO GONZÁLEZ DE REQUENA}

Las conclusiones presentadas recientemente en "What is Community Operational Research" (Midgley, Johnson y Chichirau, 2017) arrojan luces sobre la naturaleza de un debate que trasciende a su origen en Inglaterra. Al respecto, señalan que la Investigación de Operaciones Comunitaria es "el involucrarse de forma significativa con las comunidades". La expansión experimentada en lo que al principio eran las organizaciones no gubernamentales y de las comunidades organizadas sugieren que el actor se ha configurado de un modo tan complejo que, en lugar de concentrarse en el objeto de intervención, se opta por atender el modo en que se opera.

El desplazamiento hacia el tratamiento de la comunidad en términos de lo "significativo" es entendido como la participación de la comunidad en cualquiera de las etapas de formulación, identificación y solución de la situación problemática. Este planteamiento privilegia el modo como se incorpora la individualidad para ser parte de un proceso "técnico" de toma de decisiones colectivo. No hay en este modo "significativo" una noción de comunidad, aunque es de suponer que, al involucrarse en los asuntos que le conciernen de algún modo, es co-partícipe en el proceso de constituirse como sujeto. Pero, al mismo tiempo $-\mathrm{y}$ acaso porque es necesario indicarlo ya-, también se encuentra sujeto/sometido a un régimen de verdad definido en los términos de involucrarse establecidos en la Investigación de Operaciones como disciplina. El campo de las fuerzas convergentes en un proceso de gestión comunitaria deviene así en un espacio, en el cual los individuos que son la fuente para conducir la labor de preguntarse por las salidas óptimas para una determinada situación terminan constituyéndose en el vehículo para materializar que esas salidas sean viables, factibles y racionales, apelando a algo que ha quedado oculto: la aplicación de los métodos, técnicas y estrategias propias de la gestión. Esta "universalización" de las técnicas permiten diluir al sujeto dentro de los procedimientos que se suponen están validados, de forma que la singularidad que podría suponerse radica en que la organización comunitaria queda suspendida.

Sin embargo, eso es tan solo una parte de un devenir que mostraría cómo se configura a quien debe servirse de la técnica como un nodo en una red en la cual las decisiones y las racionalidades que la gobiernan están lejos de ser aquellas definidas por los actores emergentes, en este caso, las comunidades. El espectro de posibilidades asociada a la IOC ha 


\section{ONTOTECNIA, INGENIERÍA ORGANIZACIONAL Y ACTORES EMERGENTES}

sido, en este sentido, problemático y contiene en su propia práctica y en las redes de investigadores el debate sobre la "politización de la disciplina" o "el disciplinamiento de la política". De ese modo, es interesante cómo el núcleo de la práctica parece desplazarse del ámbito de la eficiencia y de la capacidad de respuesta técnica a una noción que apela a un contexto de significado no asociado al entramado tecnológico que se supone permite y sostiene la práctica de decidir en el presente.

La aparición de un afán interpretativo - aunque amenazado por la construcción de consensos- constituye uno de los aspectos sobre los cuales la IOC ha sido particularmente relevante para la reconstitución de vínculos comunitarios o, en algunos casos, de resolución de conflictos sin pérdida de las diferencias que se pueden dar entre las racionalidades de grupos marginados, etnias o cualquier otra manifestación de las formas atípicas asumidas por de las dinámicas de las decisiones en relación con la conducción de lo público en las democracias liberales.

En la actualidad, la IOC se orienta a una práctica comprometida y "encarnada" en la comunidad que es, además, de objeto de atención, sujeto activo en la construcción de los modos de resolver sus problemas o situaciones problemáticas. La IOC aparece en una relación entre iguales, en la cual la misma noción de situación problemática es definida por procesos de acompañamiento para revelar y develar las distintas racionalidades que conviven en ese proceso.

La intervención deja de ser la aplicación o inserción en la red de dispositivos para la toma de decisiones racionales, para constituirse en una construcción ad hoc de un modo de entender los propios límites de los espacios de influencia y de decisión de los distintos actores que son relevantes para enfrentar una situación que se asume compleja desde su propia definición. Así pues, es viable plantear que en el seno mismo de la práctica y concepción de la IOC hay un debate que revela una ontotecnia, la cual condiciona a los actores políticos a seguir los mecanismos de decisión amparados en la tecnología; pero, por otra parte, sugiere la posibilidad de plantear un modo alternativo de construcción de soluciones para las comunidades que tengan a la tecnología como vehículo para su construcción y legitimación en una sociedad cada vez más condicionada por lo tecnológico. 


\section{ALEJANDRO OCHOA Y JUAN ANTONIO GONZÁLEZ DE REQUENA}

En conclusión, bien pudiera plantearse que de cara a los modos de ser que se dan en el presente tecnológico hay atisbos que sugieren, si no una salida alternativa, por lo menos la posibilidad de esbozar y caracterizar algunos límites de esos modos de asumir que somos esencialmente nodos de una red, activada y desactivada en función de algunos estímulos cuyo origen no solo se desconocen, sino que incluso se opta por naturalizarlos.

Una última reflexión en torno a este modo de asumir la tecnología de decisiones para actores alternos se refiere a la necesidad de una mirada sistémica crítica, la cual sea capaz no solo de criticar los procesos de imposición de regularidades funcionales en todos los espacios de interacción social, sino que además se permita la posibilidad de cuestionar los límites esbozados en torno a la conveniencia de los distintos complejos socio-técnicos, asumidos como totalidades; ello involucra tanto la pregunta del diseño como la indagación de las condiciones que han hecho posible que ese diseño tome esas particulares características (ver Fuenmayor, 1990). Develar el carácter contingente de la propia génesis de los procesos tecnológicos puede ser un paso en el proceso de despojar a la ontotecnia de ese carácter de totalidad que recrea la sacralización del ser ahora emergiendo de los dispositivos.

\section{Conocimiento como objeto de ingeniería: Tecnología libre.}

La apuesta por una sociedad cuya construcción y desarrollo se da desde la tecnología deja de lado aspectos vinculados a la puesta en paréntesis de los modos en que las diferentes sociedades se plantean su relación con el entorno y el modo como esa relación condiciona la interacción entre los diferentes actores de la sociedad. La tecnologización de la sociedad va más allá del uso de la tecnología desde una posición de privilegio y neutralidad del actor (Feenberg, 1992). En realidad, la reconstitución del actor como un constructo tecnológico en sí mismo es lo que da lugar a un proceso que, si bien técnicamente abarca a un ámbito muy específico del conocimiento, tiene impacto más allá de lo técnico; así, la ingeniería del conocimiento va más allá del diseño de los modos de gestión de datos e información. El proceso ha alcanzado niveles en los cuales la gestión de conocimiento supone un proceso que se puede diseñar, implantar y desarrollar sin la intervención activa de la capacidad humana. Es decir, el conocimiento queda separado o al menos se lo asume independiente de un sujeto que sea capaz de ordenar, enriquecer y contextualizar. El conocimiento ha devenido no solo un bien intangible, sino además localizado fuera del ser humano y que aún sigue siendo 


\section{ONTOTECNIA, INGENIERÍA ORGANIZACIONAL Y ACTORES EMERGENTES}

considerado significativo. El conocimiento deviene en un objeto de ingeniería y, en esa misma medida, queda fuera del ser humano como su gestor y garante. Pero, ¿qué rol juega el ser humano en este proceso?

Más allá de la aproximación que pudiéramos hacer del usuario que se beneficia de la tecnología - y que es un esquema que ha servido para justificar y hacer legítimos los procesos de tecnificación de prácticamente todos los espacios de la actividad humana-, no es menos cierto que el ser humano deviene ahora en un dispositivo más incorporado a la red de dispositivos tecnológicos (Latour, 2008). Lo que esto supone en su concepción más básica es no solo la instrumentación del ser humano, sino además su carácter de accesorio y de aparición sostenida solamente sobre la condición de la conveniencia en una red de dispositivos. La técnica se hace gobierno de los modos como se revela el ser de los distintos dispositivos, incluyendo al ser humano que se supone es quien está a cargo.

Esta forma de presentar al ser humano como un nodo más en los dispositivos tecnológicos para el conocimiento va desde los aportes de la experticia cotidiana y conocimiento popular (informantes de primer nivel); incluye a quienes se encargan de procesar y sistematizar los datos en información que lo constituyen en nodos capaces de producir síntesis que podríamos denominar información, y luego a aquellos otros capaces de comprender los contextos para que esa información se convierta en conocimiento. La "tecnologización" del conocimiento es en buena medida la puesta a buen recaudo de aquello que es la competencia, por encima de la condición humana en la red de utilidades en las cuales deviene la sociedad contemporánea. La gestión del conocimiento es la gestión del talento humano que se trastoca de una gestión de la "mano de obra" a una gestión de "competencias", que van diluyendo la presencia física y desarrollando la forma virtual de estar en una red, en la cual las condiciones materiales son consideradas accesorias. Así pues, la disolución de lo material y el privilegio de lo virtual configuran un modo de ser que es esencialmente técnico. La instrumentalización que suponía un uso de una unidad denominada ser humano pierde fuerza porque lo único que se concibe como ser es aquello que se manifiesta en la red.

El modo de dar cuenta de este proceso de "virtualización" pone en el escenario la disputa entre lo que entenderíamos como un conocimiento, concebido esencialmente en un 


\section{ALEJANDRO OCHOA Y JUAN ANTONIO GONZÁLEZ DE REQUENA}

sentido no solo utilitario, sino temporal y construido sobre las redes de vinculación entre actores del conocimiento (que funcionan como simples medios para su trasmisión y la realización de una tarea específica) y, por otra parte, la aparición de espacios externos a las redes de conocimiento, en los cuales este último se constituye en un proceso social de construcción de lo común a la luz de las preguntas y las respuestas que no necesariamente respondan la forma de revelado del mundo que encarna la técnica y tecnología dominante. En este sentido, el planteamiento de la tecnología libre parece contener en sí mismo una serie elementos que pueden interrogarse desde el concepto de ontotecnia planteado.

La facilidad de acceso a la plataforma tecnológica de almacenamiento y procesamiento de información ha generado una virtualidad e integración socio-técnica de los propios dispositivos técnicos, que suponen formas de articulación de actores tecnológicos desde modalidades que son propios de las redes $\mathrm{y}$ en respuesta a intereses que no necesariamente responden a corporaciones o empresas altamente estructuradas. El ejemplo más destacado de este proceso de redes constituidas ad hoc para la producción de dispositivos tecnológicos ha sido el proyecto Linux y la explosión de las iniciativas de Software libre, y con ella las tendencias a ampliar y asegurar el acceso a la tecnología sin ningún otro requerimiento que la necesidad de utilizarla y garantizar de algún modo la sobrevivencia de la red que la propicia a través de contribuciones voluntarias. Los procesos de producción tecnológica cooperativa y colaborativa generan la posibilidad de producir tecnología de alto impacto sin la necesidad de procesos masivos de consumo de energía concentrada ni del empleo de formas coordinadas, más allá de los protocolos acordados entre los nodos de las redes dedicados a su producción.

La posibilidad de producir a muy bajo costo, de forma colaborativa, dio lugar a un proceso de hacer bien común la tecnología así desarrollada, para permitir que quien la necesita pudiera utilizarla sin que existiera ninguna restricción al respecto, salvo la de garantizar el acceso a los códigos o la apertura completa a los diseños que hacen posible el dispositivo así desarrollado.

Ante esta forma de desarrollar la tecnología intangible de las aplicaciones digitales (las tecnologías molares, esencialmente), el tema central es la apertura de todos los procesos de generación de conocimiento tal que este se convirtiera en un bien común (como se puede 


\section{ONTOTECNIA, INGENIERÍA ORGANIZACIONAL Y ACTORES EMERGENTES}

leer en el informe de Public Knowledge Project del 2018). La posibilidad de hacer del conocimiento patrimonio de la humanidad y de garantizar su acceso para quien lo necesite y no para quien lo pueda adquirir supone la reconstitución del sujeto que conoce que enfrenta la amenaza de hacer del conocimiento un bien de mercado y no de la humanidad. Si este fuese el caso, la tecnología libre estaría disputando un espacio que va a contracorriente de los modos de constitución del ser de los denominados "nativos digitales".

Aunque se considera a los procesos de incorporación masiva a los medios tecnológicos como un mecanismo de adecuación de la capacidad laboral de las masas, el abrir el espectro para la generación de tecnología a partir de las capacidades y necesidades de los diferentes actores que pueden tener acceso a la red crea un espacio de reconstitución de actores usualmente considerados como externos a las soluciones tecnológicas. Incluso, permite el desarrollo de mecanismos de indagación y respuesta entre actores que fueron desplazados de sus propias culturas y cosmovisiones. Esta posibilidad puede entenderse como una puja desde las culturas amenazadas y disminuidas, una apuesta por crear sus propias redes de constitución de significado en el espacio casi infinito generado por la diversidad de actores que hacen vida en las redes de telecomunicaciones. Sin embargo, la tendencia a abrir la tecnología a quien lo requiera $-\mathrm{y}$ desde la dimensión local de sus propias necesidades - propicia procesos de "comunalizar" la tecnología no solo en términos de hacerla situada en términos de su incorporación, sino además en su diseño y apropiación por los distintos actores en sus propios términos.

En este sentido, si bien pudiera plantearse que la ontotecnia ejerce el poder de condicionar los híbridos socio-técnicos que se adecúan al discurso hegemónico, existen manifestaciones que parecen por lo menos prolongar las posibilidades de sobrevivencia de modos alternativos de concebir la técnica y la tecnología que no se corresponden al discurso dominante de la tecnología occidental. No obstante, también puede ser el asalto definitivo para hacer que la ontotecnia adquiera la densidad suficiente para ser la única concepción desde la cual se tendrá acceso a la red.

El modo como puede entenderse el ejercicio político de los actores que practican la “comunalización" de la tecnología y la apertura a todos los seres humanos del conocimiento, considerado como un acto legítimo y políticamente apropiado en el momento que se da la 


\section{ALEJANDRO OCHOA Y JUAN ANTONIO GONZÁLEZ DE REQUENA}

mercantilización del conocimiento parece llevarse a cabo en dos planos espacio-temporales en su maduración. Un plano real construido desde la cotidianidad material caracterizada por la denuncia de un modo de ser que se considera violenta la condición humana básica de un ser que conoce y que en consecuencia está en capacidad de decidir y juzgar desde un plano político su presencia en el mundo, lo cual supondría que confronta esencialmente toda manifestación que favorezca una ontotecnia. Por otra parte, un espacio virtual que permite hacerse presentes en las formas híbridas técnico-sociales y técnico-culturales, ya no solo desde una postura de externos, sino en la capacidad concreta de ser gestores de sus propios entramados tecnológicos, tanto en la búsqueda de ser incorporados en la red de dispositivos como precisamente para denunciar su modo singular de reconstituir los sujetos despojados de la materialidad desde la cual la política se hace necesaria. Sin embargo, siempre queda pendiente la opción de que, al hacerse de la tecnología como herramienta de emancipación y reconocimiento de nuevos actores, ya no se ha dado el paso para ser un nodo más en una omniabarcante red de redes. En este caso, sería curioso que el último acto político fuese contenido en su totalidad por la virtualización de lo que es humano.

\section{Una perspectiva de conjunto de los actores ontotécnicos}

Parece existir cierta relación de isomorfismo estructural y de solidaridad funcional entre las discontinuidades de la deriva ontotécnica (de la fase tecnológica del ensamblaje mecánico, a la fase de la automotorización dinámica, a la fase de las redes comunicacionales) y, por otra parte, la irrupción de nuevos actores sociopolíticos. También la construcción de los actores sociopolíticos ha conocido una fase cinemática de agregación numérica y de integración mecánica de los individuos. La portada original del Leviathan de Hobbes ilustra bien esta problemática de la integración de un cuerpo político soberano (véase la figura 2). 


\section{ONTOTECNIA, INGENIERÍA ORGANIZACIONAL Y ACTORES EMERGENTES}

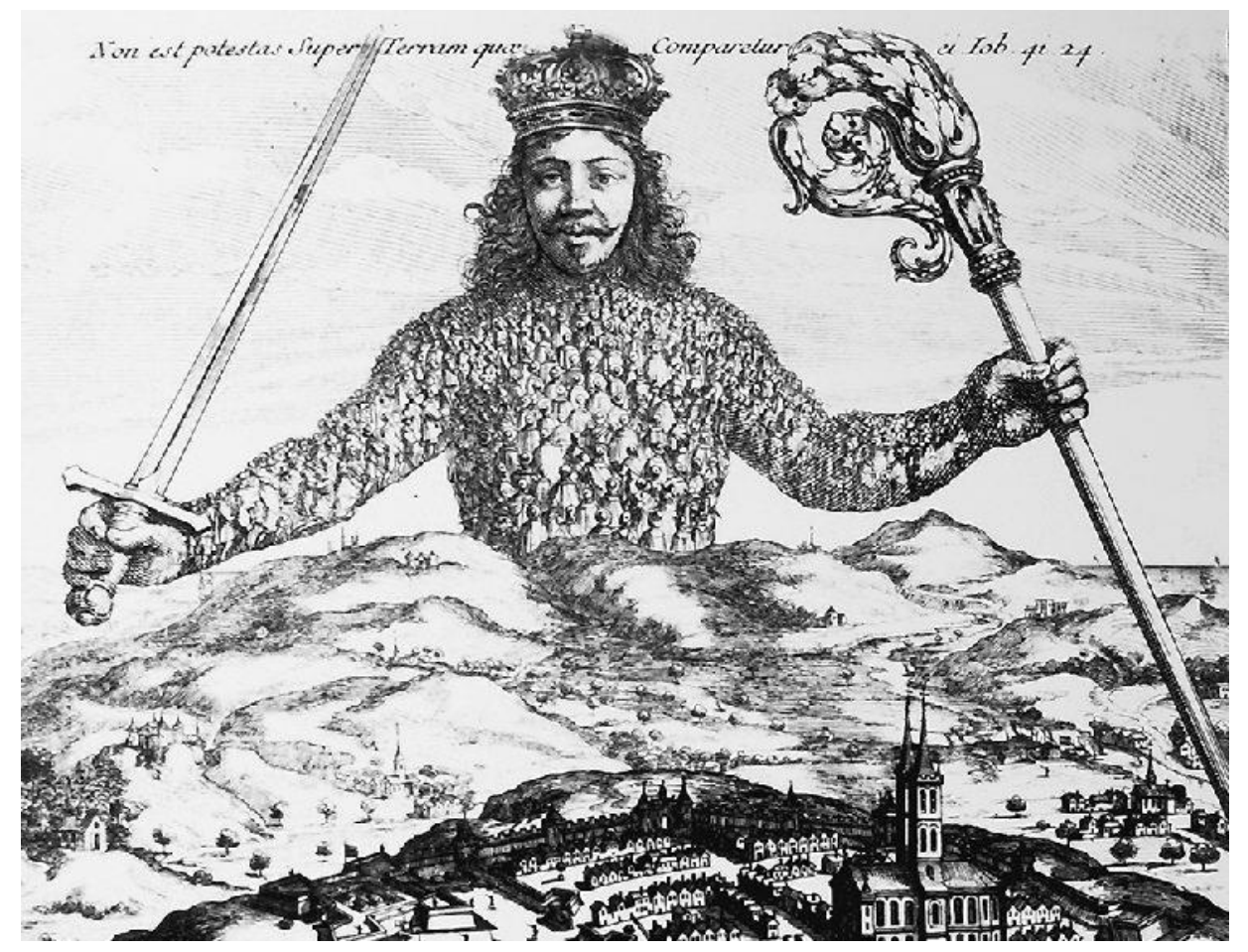

Figura 2. La integración del cuerpo político en la portada del Leviathan. Fuente: https://semanariouniversidad.com/suplementos/los-hijos-de-leviatan/attachment/1-93leviathan/

De hecho, la cuestión del número mayoritario, de la división de las partes y de la unificación de lo múltiple ha sido decisiva en la construcción sociopolítica tradicional, desde la polis griega hasta las democracias liberales modernas. La agregación numérica ha permitido organizar las formas de los regímenes políticos según el número de quienes dirigen y de quienes son dirigidos, de manera que se distingue entre el gobierno de uno, el gobierno de algunos calificados o el gobierno de los muchos. Además, la integración aritmética ha hecho posible establecer formas de representación basadas en el principio mayoritario; incluso, puede decirse que la unificación de lo múltiple bajo la figura de la unanimidad aritmética y de la integración de las diferencias permitió concebir un mecanismo de legitimación para la construcción sociopolítica. No obstante, la separación analítica de poderes o funciones políticas abstractamente autónomas ha aportado un marco formal para sobrellevar las tensiones entre la unificación sumativa de la mayoría, la unanimidad sin resta de la voluntad general soberana y, además, la división de las partes en el reparto distributivo de la geometría sociopolítica. Asimismo, el cálculo y la contabilidad racionales, así como la 


\section{ALEJANDRO OCHOA Y JUAN ANTONIO GONZÁLEZ DE REQUENA}

administración imparcial, ejercidos a través de burocracias que especializan las competencias funcionales, han posibilitado un tipo de construcción sociopolítica capaz de integrar las necesidades y de responder al interés general. También la garantía formal de la equivalencia abstracta de los individuos atomizados, es decir el derecho racional, ha contribuido a hacer de la agregación mecánica de las partes un principio de construcción sociopolítica. Por otra parte, las diferencias decisivas en la construcción sociopolítica se han inscrito fundamentalmente bajo la figura geométrica de las facciones, las partes y eventualmente los partidos; esa es, de hecho, la forma fundamental de investidura de los actores en la fase cinemática de la construcción sociopolítica: la división de facciones y partidos que pugnan por integrar una amplia mayoría representativa (Rosanvallon, 2010).

Sin embargo, la construcción sociopolítica moderna también ha atravesado una fase dinámica en que se trata fundamentalmente de canalizar orgánicamente las energías sociales y de llevar a cabo una movilización total de todos los recursos, convertidos así en variables operacionales de un proceso de transformación integral. No solo los movimientos totalitarios del siglo XX (del utopismo productivista, al modernismo reaccionario) se asocian a esta dinámica sociopolítica que pretende movilizar exhaustivamente, transformar plenamente, integrar orgánicamente y organizar íntegramente tanto las tendencias funcionales cuanto las coherencias operacionales y las energías socio-naturales disponibles. La movilización general de las fuerzas y energías productivas —incluidos los recursos humanos-constituye un vector subyacente de la construcción sociopolítica que ha orientado y conformado estructuralmente el ordenamiento político, jurídico y estatal moderno. La movilización productiva orientada a la conformación de una economía-mundo capitalista (y presupuesta por el ordenamiento político liberal de las necesidades y los intereses) implicó el enrolamiento laboral orgánico, la cualificación exhaustiva de los rendimientos y la organización de las formas de movilidad en conjuntos funcionales, desde la escuela y la ciudad, hasta la fábrica y el frente. Ya sea a través del encuadramiento disciplinario de los cuerpos y los movimientos poblacionales, de la organización panóptica de visibilidades y vigilancias, o bien del acoplamiento operacional a los ritmos funcionales de la máquina, se ha desplegado toda una dinámica socio-técnica orientada a la plena integración orgánica de los rendimientos funcionales, mediante una constante transformación productiva (de Gaudemar, 1981). 


\section{ONTOTECNIA, INGENIERÍA ORGANIZACIONAL Y ACTORES EMERGENTES}

No es de extrañar que muchos de los léxicos de la construcción sociopolítica moderna estén marcados por una semántica dinámica de la movilización, en la medida en que sus categorías aparecen temporalizadas como "ismos" y se transforman en consignas anticipadoras al servicio de la auto-movilización general futurista, legitimada como progreso (Koselleck, 1993). Asimismo, las diferencias sociopolíticas se inscriben en un léxico dinámico que opone a los activos promotores de las fuerzas de la movilización histórica, los progresistas, y, por otra parte, a los antagonistas de la automovilización futurista, los reaccionarios (Starobinski, 2001). En esta construcción sociopolítica dinámica, el actor se inscribe operacionalmente bajo la figura del movimiento, a la vez que se consolidan las formas de representación y legitimación funcionales a la automovilización, esto es, las ideologías, auténticos motores del proceso de movilización total o de una revolución permanente. Encontramos cabalmente ilustrada esta construcción del movimiento y la consagración de la movilización total en la propaganda totalitaria; por ejemplo, en la propaganda nazi (véase la figura 3).

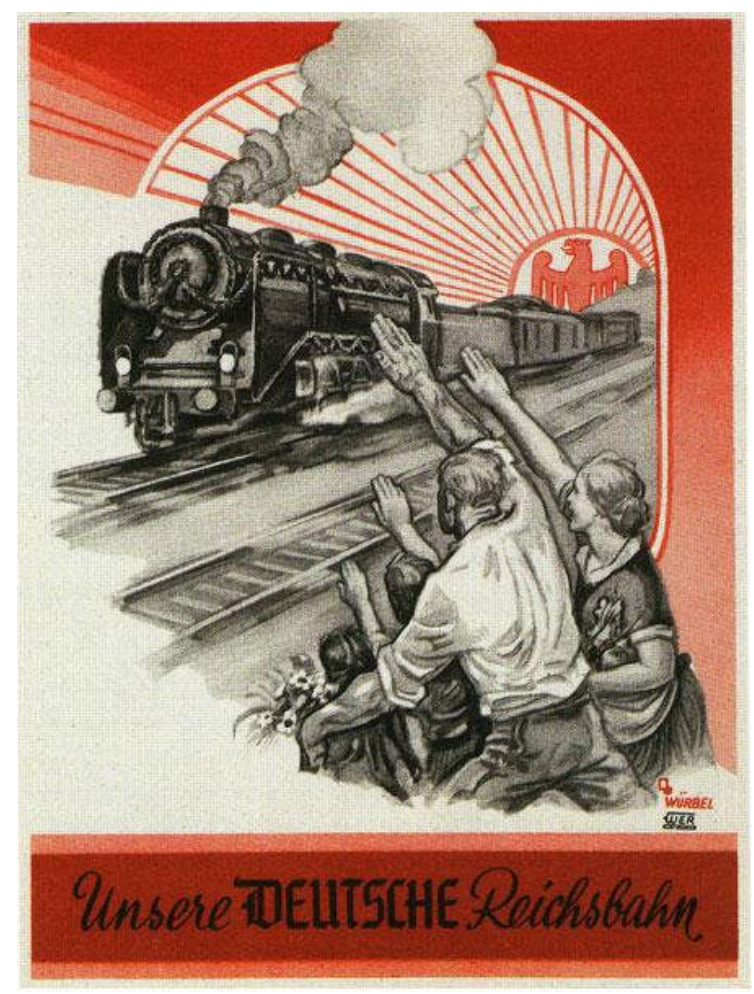

Figura 3. La movilización técnico-política en la propaganda nazi. Fuente: http://www.museumsyndicate.com/images/7/69115.jpg 


\section{ALEJANDRO OCHOA Y JUAN ANTONIO GONZÁLEZ DE REQUENA}

Cabe preguntarse, finalmente, qué figura corresponde a los actores de la construcción sociopolítica que emergen a través de las contemporáneas redes comunicacionales. Por una parte, es posible observar nuevas formas de articulación de los actores, que no dependen de la agregación o la movilización presencial de individuos y masas; se trata de una modalidad reticular y descentrada de construcción sociopolítica, en coherencia operacional con las nuevas redes telemáticas y con el creciente desanclaje de las interacciones multimediáticas, que se asocia a la transmisión de los flujos informacionales y a la interconexión en los canales virtuales de comunicación, así como a los acoplamientos interactivos con los dispositivos comunicacionales y las pantallas mediáticas. En ese sentido, las nuevas formas de interacción comunicacional y de proximidad mediática maximizan los contactos interactivos, y facultan todo tipo de tele-relaciones simultáneas y deslocalizadas, que incluso pueden alcanzar una dimensión global y virtualizar lo público.

Pero, al mismo tiempo, las tele-interacciones desplegadas en las redes comunicacionales parecen reducir la escala de los tópicos de la comunicación sociopolítica. No en vano, privilegian frecuentemente la tematización de los asuntos personales, las microsolidaridades en torno a estilos particulares de vida y a pequeños vínculos íntimos de escasa resonancia pública; aunque también potencian las formas de identificación representativa con algunos personajes mediáticamente publicitados. De esa manera, la legitimación de las construcciones sociopolíticas contemporáneas gira, en gran medida, en torno a la interactividad y apertura comunicacional, la cercanía y el carisma personales; aunque de ello se desprenda el riesgo de una mediatización y espectacularización de la razón pública (Rosanvallon, 2010).

En efecto, con frecuencia, se han señalado los riesgos de cierta deriva hacia una teledemocracia y hacia una sociedad del espectáculo, que hiperrealiza mediáticamente nuestra construcción sociopolítica, hasta el punto de suplantarla finalmente y simularla indefinidamente; todo ello, mediante la circulación masiva de simulacros interactivos y la conexión indiferente a la interfaz, de tal manera que se consumaría plenamente la inversión de lo real y la mediatización imaginaria (Baudrillard, 1993; Debord, 2002). Se trata, en todo caso, de una interpretación bastante unilateral de las interacciones mediáticas, que omite la actividad social de la recepción situacional, la apropiación cotidiana rutinaria y el proceso de 


\section{ONTOTECNIA, INGENIERÍA ORGANIZACIONAL Y ACTORES EMERGENTES}

interpretación de los mensajes mediáticos, el cual requiere movilizar numerosos atributos sociales y competencias cognitivas (Thompson, 1998).

La construcción sociopolítica en las redes mediáticas contemporáneas también abre la posibilidad de conformar nuevos espacios públicos descentrados y formas de aparición en público no estatales, que se basarían en la constitución de redes de arquitectura abierta y de difícil control, en la difusión sin restricciones de la información, en la visibilización de nuevos temas en la agenda pública, así como en la concurrencia innovadora de la inteligencia colectiva. En el contexto de una construcción sociopolítica mediática, operacionalmente articulada a través de las redes comunicacionales, se podría concluir, pues, que los actores emergentes se inscriben y se exponen como públicos —a menudo contraculturales-, esto es, como múltiples comunidades imaginarias, discursivamente e icónicamente autoorganizadas a través de las redes mediáticas, que reescenifican performativamente los estilos de pertenencia y los guiones de las solidaridades sociales (Warner, 2002).

\section{En conclusión: la espectralidad y el sentido político de los actores emergentes}

Aun cuando en las redes mediáticas parecen conformarse modalidades de construcción sociopolítica caracterizadas por la multiplicación de los públicos, seguimos recurriendo al léxico dinámico tardomoderno y designamos a los actores emergentes como nuevos movimientos sociales. Se continúa apelando a los movimientos sociales, aun cuando los actores emergentes no respondan a las características de aquellos actores que se autodesignaron dinámicamente como movimientos. No en vano, los actores emergentes eluden las formas de movilización permanente y la plena participación en las dinámicas de conjunto de las fuerzas sociales. Además, se rechazan los guiones funcionales de conjunto y las codificaciones ideológicas de los conflictos sociopolíticos, así como se dispersan las formas de resistencia a través de asuntos públicos y privados fragmentarios, sin que se conformen organizaciones estables y centralmente coordinadas. Las modalidades de interacción y participación de los actores emergentes parecen vincularse en gran medida a la "movilización cognitiva" y al desarrollo de competencias de articulación de demandas, así como a la actividad autorreferente y espontáneamente autoorganizada en una multiplicidad de nodos y redes. Así, pues, los actores emergentes no persiguen la toma del poder ni la movilización total o la coordinación de un único movimiento de conjunto (von Beyme, 1994). 


\section{ALEJANDRO OCHOA Y JUAN ANTONIO GONZÁLEZ DE REQUENA}

El hecho de que sigamos designando como movimientos sociales a estos actores emergentes concierne a la espectralidad de toda construcción ontotécnica, en virtud de la cual, las configuraciones socio-técnicas que irrumpen no pueden dejar de reproducir la investidura simbólica y los léxicos prestados de construcciones anteriores. Sin embargo, los léxicos con que categorizamos a estos actores emergentes en ningún caso describen aquello que alguna vez significaron. Únicamente refieren el fantasma de las configuraciones sociotécnicas desbordadas: cierta rémora espectral reproducida bajo las condiciones de un nuevo modo de construcción socio-técnica, y que invoca más que nada un deseo imaginariamente proyectado. Así como los movimientos políticos tardomodernos se autoinvistieron fantasmáticamente con el léxico clásico de los partidos, actualmente nombramos a los públicos emergentes de las redes mediáticas, conjurando el espectro de los movimientos sociales.

No se ha de concluir de nuestra fantasmología ontotécnica de los actores emergentes que las nuevas formas de subjetivación, interacción y participación políticas estén determinadas de alguna manera por las "olas" o los "hados" de alguna versión de determinismo tecnológico. Nuestra descripción de ciertos isomorfismos entre la construcción ontotécnica y la construcción sociopolítica tampoco ha de conducir a cierta vulgata construccionista que sostiene que el sentido político de los actores emergentes surge de alguna forma de co-construcción o de co-determinación recíproca de ambos medios constructivos. El sentido y la eficacia políticos de los actores emergentes no emerge de alguna construcción reflexiva y recursiva de los entornos socio-técnicos, sino de la falla que atraviesa todo intento de distribuir las partes y organizar conjuntamente los componentes de la construcción. Se trata de esa falla que atraviesa espectralmente toda construcción sociotécnica, en la medida en que sus formatos de construcción no pueden eludir el desfase fantasmático de los modos de reconocimiento imaginario que lo acompañan. Lo político de los actores emergentes actúa allí donde las formas de reconocimiento imaginario y los espectros conjurados desmantelan toda clausura de la construcción socio-técnica; allí donde irrumpen fantasmáticamente las demandas invocadas de las partes que no se reconocen como parte del orden socio-técnico. 


\section{ONTOTECNIA, INGENIERÍA ORGANIZACIONAL Y ACTORES EMERGENTES}

\section{Referencias}

Baudrillard, J. (1993). La transparencia del mal. Barcelona: Anagrama.

Benjamin, W. (1973). Discursos interrumpidos I. Madrid: Taurus.

Debord, G. (2002). La sociedad del espectáculo. Valencia: Pre-textos.

Debray, R. (2001). Introducción a la mediología. Barcelona: Paidós.

Derrida, J. (1995). Espectros de Marx. Madrid: Editorial Trotta.

de Gaudemar, J. P. (1981). La movilización general. Madrid: La Piqueta.

Feenberg, A. (1992). Subversive Rationalization: Technology, Power and Democracy. Inquiry, 35 (3-4), 301-322.

Fuenmayor, R. (1990). Systems Thinking and Critique I. What is Critique?. Systems Practice and Action Research, 3 (6), 525-544

Gadamer, H. G. (1998). El giro hermenéutico. Madrid: Cátedra.

Haraway, D. (1991). Simians, Cyborgs, and Women. New York: Routledege.

Heidegger, M. (1990). Identidad y Diferencia. Barcelona: Anthropos.

Heidegger, M. (1991). La proposición del fundamento. Barcelona: Ediciones del Serbal.

Heidegger, M. (1994). Conferencias y artículos. Barcelona: Ediciones del Serbal.

Heidegger, M. (1996). Caminos del bosque. Madrid: Alianza Editorial.

Johnson, M.P. (2012). Community-based operations research: Introduction, theory and applications. En M.P. Johnson (Ed.), Community-based operations research: Decision modeling for local impact and diverse populations (pp. 3-37). New York. Springer.

Koselleck, R. (1993). Futuro pasado. Barcelona: Paidós.

Latour, B. (2007). Nunca fuimos modernos. Buenos Aires: Siglo XXI.

Latour, B. (2008). Reensamblar lo social: Una introducción a la teoría del actor-red. Buenos Aires: Manantial.

Lévy, P. (2004). La inteligencia colectiva. Por una antropología del ciberespacio. Washington D.C.: Organización Panamericana de la Salud.

McLuhan, M. (1985). La galaxia Gutenberg. Barcelona: Planeta-Agostini.

McLuhan, M. y Powers, B. R. (1996). La aldea global. Barcelona: Gedisa.

Midgley, G. (2000). Systemic Intervention: Philosophy, methodology and Practice. NewYork. Kluwer/Plenum 


\section{ALEJANDRO OCHOA Y JUAN ANTONIO GONZÁLEZ DE REQUENA}

Midgley, G., Johnson, M. y Chichirau, G. (2017). What is Community operational Research? European Journal of Operational Research. 1-13

Midgley, G. y Ochoa Arias, A (1999). Visions of community for community OR. Omega, 27, 259-274.

Midgley, G., y Ochoa Arias, A. (eds.) (2004). Community Operational Research: OR and Systems thinking for community development. New York, Kluwer.

Mumford, L. (1987). Técnica y civilización. Madrid: Alianza Editorial.

Parry, R., y Mingers, J. (1991). Community Operational Research: Its context and its future. Omega, 19, 577-586.

Poe, E. A. (1973). Ensayos y críticas. Madrid: Alianza Editorial.

Ritchie, C.; Taket, A. y Bryant, J. (Eds.). (1994). Community works: 26 case studies showing community operational research in action. Sheffield: Pavic Press.

Rorty, R. (1991). Contingencia, ironía y solidaridad. Barcelona: Paidós.

Rosanvallon, P. (2010). La legitimidad democrática. Barcelona: Paidós.

Rosenhead, J. (1986). Custom and Practice. Journal of the Operational Research Society, 37, $335-343$

Serres, M. (1995). Atlas. Madrid: Cátedra.

Simondon, G. (2007). El modo de existencia de los objetos técnicos. Buenos Aires: Prometeo.

Starobinski, J. (2001). Acción y reacción. México D. F.: Fondo de Cultura Económica.

Stiegler, B. (2002). La técnica y el tiempo I. Gipuzkoa: Editorial Hiru.

Thompson, J. B. (1998). Los media y la modernidad. Barcelona: Paidós.

Vattimo, G. (1991). Ética de la interpretación. Barcelona: Paidós.

Von Beyme, K. (1994). Teoría politica del Siglo XX. De la modernidad a la postmodernidad. Madrid: Alianza Editorial.

Warner, M. (2002). Publics and Counterpublics, Public Culture, 14 (1), 49-90. 


\section{REVISTA STVLTIFERA DE HUMANIDADES Y CIENCIAS SOCIALES}

VOLUMEN 1, NÚMERO 1, PRIMER SEMESTRE DEL 2018

ISSN 0719-983X

\section{Artículos de Humanidades y Ciencias Sociales}

La violencia como espectáculo.

Juan Carlos Pérez Jiménez

El amo castrado.

Elisa Freijo Corbeira

España, una meditación política: Cataluña y Euskadi.

Mikel Aramburu Zudaire

Del saber sabio al saber dramatizado. Nuevos fundamentos para la aplicación permanente del teatro en el aula.

Luis Fernando Lara Coronado

Ontotecnia, ingeniería organizacional y actores emergentes.

Alejandro Ochoa Arias y Juan Antonio González de Requena Farré

\section{Reseñas}

Memoria Visual de Legua Emergencia, vida y oficio de Mario Alarcón.

Pedro Pablo Achondo Moya

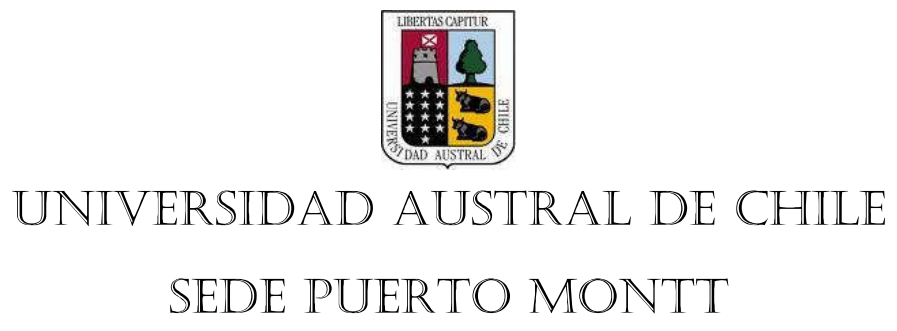

